\title{
The Role of Notch1 Signaling in Anaplastic Thyroid Carcinoma
}

Hyeon Jin Kim, $\mathrm{MS}^{1}$
Min-Jung Kim, $\mathrm{PhD}^{1}$
Areumnuri Kim, $\mathrm{PhD}^{1}$
Chang Won Jung, $\mathrm{MD}^{2}$
Sunhoo Park, $\mathrm{MD}, \mathrm{PhD}^{1,2}$
Jae Soo Koh, MD, $\mathrm{PhD}^{2}$
Jae Kyung Myung, $\mathrm{MD}, \mathrm{PhD}^{1,2}$

${ }^{1}$ Laboratory of Radiation Pathology, ${ }^{2}$ Department of Pathology, Korea Cancer Center Hospital, Seoul, Korea
Correspondence: Jae Kyung Myung, MD, PhD Department of Pathology, Korea Cancer Center Hospital, 75 Nowon-ro, Nowon-gu,

Seoul 01812, Korea

Tel: 82-2-970-1257

Fax: 82-2-970-2430

E-mail: tontos016@naver.com

Received May 24, 2016

Accepted August 12, 2016

Published Online September 1, 2016

\section{Purpose}

The Notch signaling pathway is widely expressed in normal, reactive, and neoplastic tissues; however, its role in thyroid tissues has not been fully elucidated. Therefore, this study was conducted to characterize the expression of the Notch signaling pathway in papillary thyroid cancer (PTC) cells and anaplastic thyroid cancer (ATC) cells.

\section{Materials and Methods}

Expression of activated Notch1 in ATC and PTC paraffin-embedded tissues was determined by immunohistochemistry. The small interfering RNA technique was employed to knock down Notch1 expression in ATC and PTC cell lines.

\section{Results}

The expression of activated Notch1 was higher in ATC cases than in PTC cases. Inhibition of Notch1 significantly reduced proliferation and migration of ATC cells, but not PTC cells. In addition, inhibition of Notch1 in ATC cells significantly reduced the expression of key markers of epithelial-mesenchymal transition and cancer stem cells. Conversely, changes in the expression of these proteins were not observed in PTC cells.

\section{Conclusion}

The results of this study suggest that Notch1 expression plays different roles in tumor progression in ATC and PTC cells. We also found that Notch1 expression was significantly related to the highly invasive or proliferative activity of ATC cells.

\section{Introduction}

Thyroid cancer is one of the most common endocrine malignancies, and its incidence continues to rise [1]. Thyroid cancers have been divided into several representative subtypes according to histopathological features; namely, papillary, follicular, medullary, and anaplastic thyroid carcinoma (ATC) [2]. ATC, an undifferentiated tumor of the thyroid, has the most aggressive clinical behavior because of its

\section{Key words}

Receptor Notch1, Anaplastic thyroid carcinoma, Papillary thyroid cancer, Epithelial-mesenchymal transition, Neoplastic stem cells 
patients. In this study, we focused on the Notch signaling pathway as a therapeutic target. Notch is a multifunctional transmembrane receptor that regulates cellular differentiation, development, proliferation, and survival of normal or cancer cells in a variety of contexts [6,7]. Notch signaling is made up of four Notch receptors (Notch1, 2, 3, and 4) and five ligands (Delta 1, 3, and 4 and Jagged [Jag] 1 and 2) in humans [8]. Binding of any one of the Notch ligands to the receptor promotes two proteolytic cleavages, which are mediated by ADAM metalloprotease and $\gamma$-secretase enzymes. This results in release of the Notch intracellular domain (NICD) from the cell transmembrane and its subsequent translocation into the nucleus [9-12]. The Notch signaling pathway affects the progression of several cancers [13-16]. Indeed, previous studies have suggested that Notch1 could play a key role in the regulation of epithelial-mesenchymal transition (EMT) and cancer stem cell (CSC) phenotype during the development and progression of tumors $[17,18]$. However, the role of Notch signaling in thyroid cancer is poorly elucidated. Therefore, we evaluated the role of Notch signaling using tissue samples from ATC and papillary thyroid carcinoma (PTC). In addition, we examined the cell proliferation and migration of ATC and PTC cells after knockdown of Notch1 signaling and evaluated alterations in protein expression related to EMT or CSC.

\section{Materials and Methods}

\section{Tissue samples and immunohistochemistry}

Paraffin blocks and slides from 16 cases of ATC and 109 cases of PTC (between 1998 and 2013) were retrieved from the archives of the Department of Pathology at the Korea Cancer Center Hospital. The electrical medical records of the patients corresponding to these cases were reviewed. This study was approved by the Institutional Review Board of the Korea Cancer Center Hospital.

Immunohistochemical staining was conducted by permeabilizing with $0.1 \%$ Triton X-100 in phosphate buffered saline (PBS) for 10 minutes, quenching endogenous peroxides with $3 \%$ hydrogen peroxide for 10 minutes, blocking, and then incubating the samples with primary and secondary antibodies. Immunoreactivity was visualized with avidin-biotin-peroxidase. The primary antibody for activated Notch1 was purchased from Abcam (Cambridge, UK) and used at a dilution of 1:200.

Pearson's chi-squared test was used to assess the relevance of expression of activated Notch1 when comparing ATC and PTC cases. Statistical analyses were performed using SPSS ver. 18 (SPSS Inc., Chicago, IL) and a $\mathrm{p}<0.05$ was considered significant.

\section{Cell cultures}

Human papillary (BCPAP) and anaplastic (8505C) thyroid cancer cells were provided by Dr. Min Joo Kim (Korea Cancer Center Hospital, Seoul, Korea). The BCPAP cell lines were cultured in RPMI-1640 (Lonza, Wakersville, MD), and 8505C cell lines were maintained in Dulbecco's modified Eagle medium (WelGENE Inc., Daegu, Korea) supplemented with 10\% fetal bovine serum (FBS; JR Scientific Inc., Woodland, CA) and $0.1 \%$ gentamycin (Lonza) at $37^{\circ} \mathrm{C}$ in $5 \% \mathrm{CO}_{2}$.

\section{Small interfering RNA transfection}

Small interfering RNAs (siRNAs) against Notch1 and scrambled siRNA (siScr) used as a negative non-silencing control were synthesized by Genolution Pharmaceuticals (Seoul, Korea). The following siRNA sequences were used in this study: Notch1 \#1, 5'-GCGACAAGGUGUUGACGUU-3'; Notch1 \#2, 5'-GAUGCGAGAUCGACGUCAA-3'; and siScr, 5'-UGGUUUACAUGUCGACUAA-3'. Cells were cultured on $60-\mathrm{mm}$ dishes to $50 \%-75 \%$ confluence, then transfected with 50 nM siRNA using Lipofectamine 2000 (Invitrogen, Carlsbad, CA) according to the manufacturer's instructions.

\section{Cell proliferation assay}

The effects of Notch1 on thyroid cancer cell proliferation were tested by counting the number of viable cells. The cells were seeded at a density of $1 \times 10^{5}$ cells in 60 -mm dishes and transfected with siNotch1 using Lipofectamine 2000. After a 72-hour incubation period, the number of viable cells was counted using an ADAM cell counter (NanoEnTek, Seoul, Korea) according to the manufacturer's instructions. Percent viability was determined by comparison with siScr-transfected control samples.

\section{Propidium iodide uptake assay}

The effects of Notch1 on thyroid cancer cell death were determined using propidium iodide (PI; Sigma, St. Louis, MO) uptake. The cells were seeded at a density of $1 \times 10^{5}$ cells in 60-mm dishes and transfected with siNotch1 for 72 hours. After washing the cells with PBS, PI solution was immediately added. After staining, the cells were analyzed using a FACS Canto II flow cytometer (BD Biosciences, San Diego, CA). 


\section{Migration assay}

The effect of Notch1 on thyroid cancer cell migration ability was assessed using $8-\mu \mathrm{m}$ pore size transwell chambers (Corning Life Science, Corning, NY). Briefly, $3 \times 10^{4}$ cells transfected with siScr or Notch1 siRNA in serum-free medium were plated in the upper chamber. Medium containing $10 \%$ FBS was added to the lower compartment of the chamber. After 48 hours of incubation, the cells were fixed with $4 \%$ paraformaldehyde and stained with crystal violet. The migrated cells in three randomly selected fields for each well were visualized with an Olympus BX53F microscope and cellSens imaging software (Olympus, Tokyo, Japan) using a $100 \times$ objective.

\section{7. $\gamma$-Secretase inhibitor treatment}

$\mathrm{N}$-[N-(3,5-Difluorophenacetyl)-L-alanyl]-S-phenylglycine $t$-butyl ester (DAPT; Sigma), a $\gamma$-secretase inhibitor, was used to block the Notch signaling pathway. DAPT was dissolved in dimethyl sulfoxide at a concentration of $100 \mathrm{mM}$. Cells were seeded at a density of $1 \times 10^{5}$ per $60-\mathrm{mm}$ dish and treated with 0 , 2, or $5 \mu \mathrm{M}$ DAPT. After incubation for 72 hours, the cells were analyzed by proliferation and migration assays according to the manufacturer's protocols.

\section{Western blotting analysis}

Whole cell lysates and nuclear proteins were extracted using NP-40 Protein Extraction Solution (Invitrogen) according to the manufacturer's instructions. Whole cell lysates (30 $\mu \mathrm{g}$ protein per lane) were separated by $8 \%$ or $12 \%$ sodium dodecyl sulfate-polyacrylamide gel electrophoresis. The separated proteins were then electrophoretically transferred onto polyvinylidene fluoride membranes and incubated with primary antibodies overnight at $4^{\circ} \mathrm{C}$. Immunoreactive bands were then developed using a horseradish peroxidase-conjugated secondary antibody, and the proteins were visualized

A
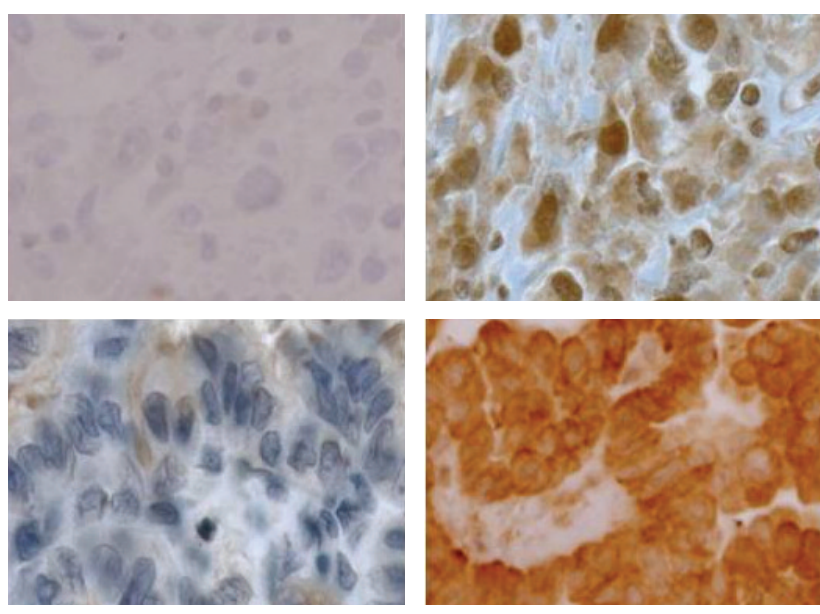

\begin{tabular}{llccc} 
& & ATC & PTC & p-value \\
Activated Notch1 & Negative & $2(12.5 \%)$ & $82(75.2 \%)$ & $<0.001$ \\
& Positive & $14(87.5 \%)$ & $27(24.8 \%)$ & \\
Total & & $16(100 \%)$ & $109(100 \%)$ & \\
\hline
\end{tabular}
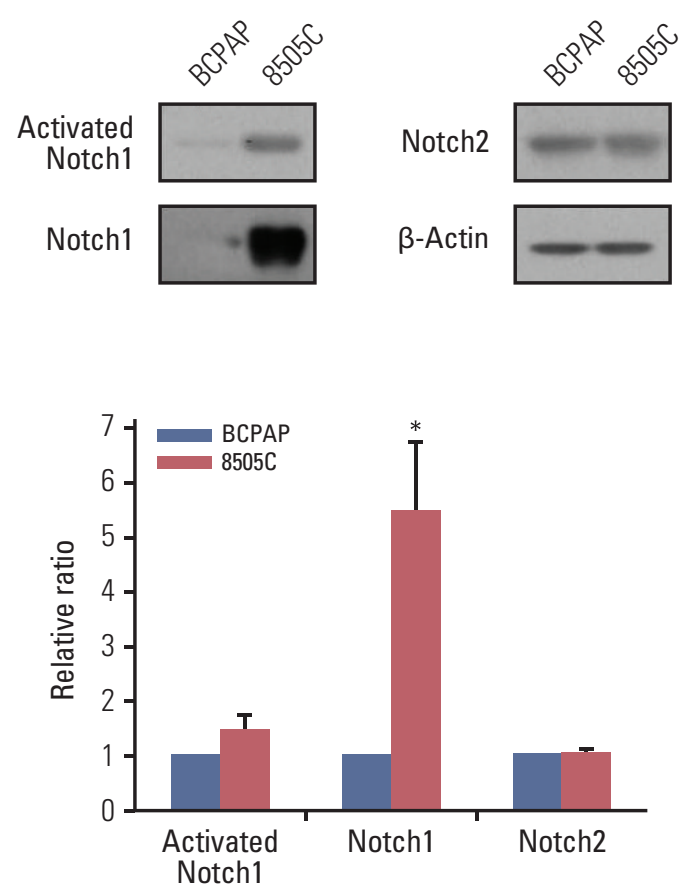

Fig. 1. Expression of activated Notch1 in patient tissues and thyroid cancer cell lines. (A) Immunohistochemical analysis of activated Notch1 expression in anaplastic (upper left, negative; upper right, positive; $\times 400$ ) and papillary (lower left, negative; lower right, positive; $\times 400$ ) thyroid cancer tissues. (B) Western blot analysis of activated Notch1, Notch1, and Notch2 expression in BCPAP and 8505C thyroid cancer cell lines. Each graph shows the target protein expression ratio normalized to $\beta$-actin. Statistical significance is based on the difference when compared with BCPAP cells, ${ }^{*} \mathrm{p}<0.05$. ATC, anaplastic thyroid cancer; PTC, papillary thyroid cancer. 
A

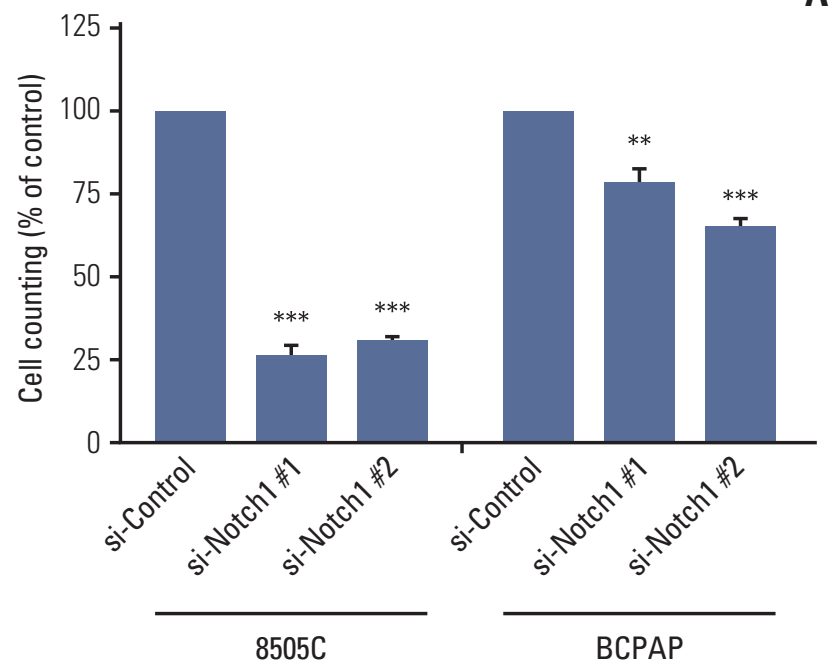

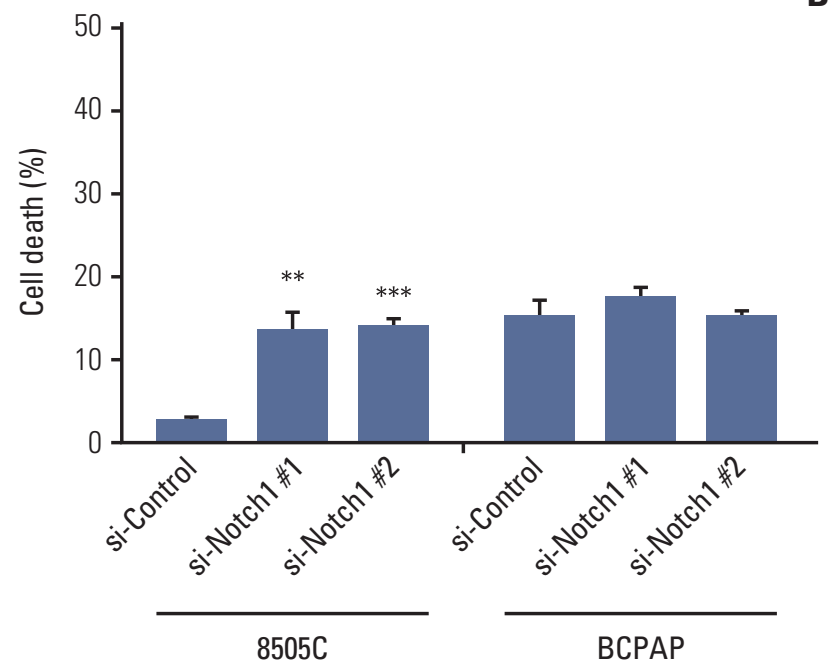

Fig. 2. Knockdown of Notch1 reduced cell viability in anaplastic thyroid cancer cell lines. 8505C and BCPAP cells were transfected with si-control or si-Notch1 for 72 hours, after which proliferation was determined by cell counting (A) and propidium iodide uptake (B). Statistical significance is based on the difference when compared with si-control cells, ${ }^{* *} \mathrm{p}<0.01$, ${ }^{* * *} \mathrm{p}<0.001$.

by enhanced chemiluminescence (Amersham Biosciences, Piscataway, NJ) using the manufacturer's protocols. The following primary antibodies were used: Notch1, Notch2, Musashi, and Snail (Cell Signaling Technology Inc., Danvers, MA); activated Notch1 and activated Notch2 (Abcam, Cambridge, MA); E-cadherin and N-cadherin (BD Biosciences); Sox2, Oct3/4, Nanog, and $\beta$-actin (Santa Cruz Biotechnology, Santa Cruz, CA).

\section{Statistical analysis}

Values are expressed as the means \pm standard error of mean of the results of at least three experiments. The values were evaluated by Student's t test using the GraphPad Prism 4.0 software (GraphPad Software, San Diego, CA). Differences with $p$-values of $<0.05$ were considered statistically significant.

\section{Results}

\section{Expression of activated Notch 1 in thyroid cancer patient tissues and thyroid cancer cell lines}

We examined the activated Notch1 (NICD) protein expression as an indication of the activation of Notch1 signaling in
ATC and PTC patient tissues using immunohistochemistry. Immunoreactivity in the nucleus and cytoplasm was regarded as positive staining. As shown in Fig. 1A, we found that activated Notch1 was strongly expressed in more ATC cases than PTC cases. Different expression of activated Notch1 between ATC and PTC cases was statistically significant $(\mathrm{p}<0.001)$.

We analyzed the basal levels of Notch1, Notch2, and activated Notch1 protein expression in $8505 \mathrm{C}$ and BCPAP cells. Notch1 and activated Notch1 proteins were more highly expressed in $8505 \mathrm{C}$ cells than in BCPAP cells, but Notch2 protein levels were not (Fig. 1B).

\section{Knockdown of Notch1 reduced cell viability in ATC cell lines}

To investigate the effects of Notch1 signaling on cell viability and cell death, 8505C and BCPAP cells were transfected with Notch1 siRNA at $50 \mathrm{nM}$ for 72 hours. As shown in Fig. 2, knockdown of Notch1 significantly inhibited cell viability in both $8505 \mathrm{C}$ and BCPAP cells. In addition, inhibition of Notch1 increased cell death in $8505 \mathrm{C}$ cells when compared to cells treated with control siRNA. However, this effect was not found in BCPAP cells. These results showed that knockdown of Notch1 could reduce the cell viability of ATC cells. 
$8505 C$

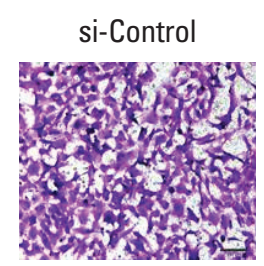

\section{si-Notch1 \#2}

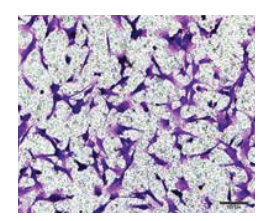

\section{si-Notch1 \#2}

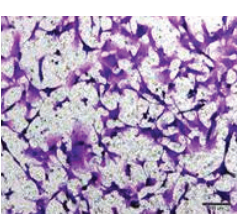

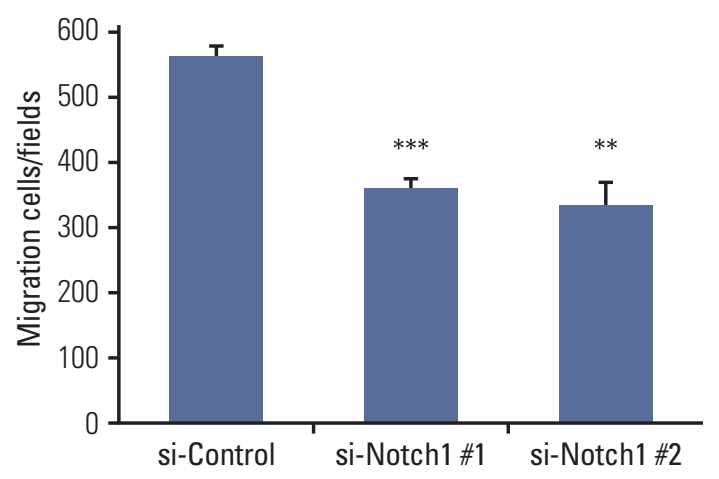

BCPAP
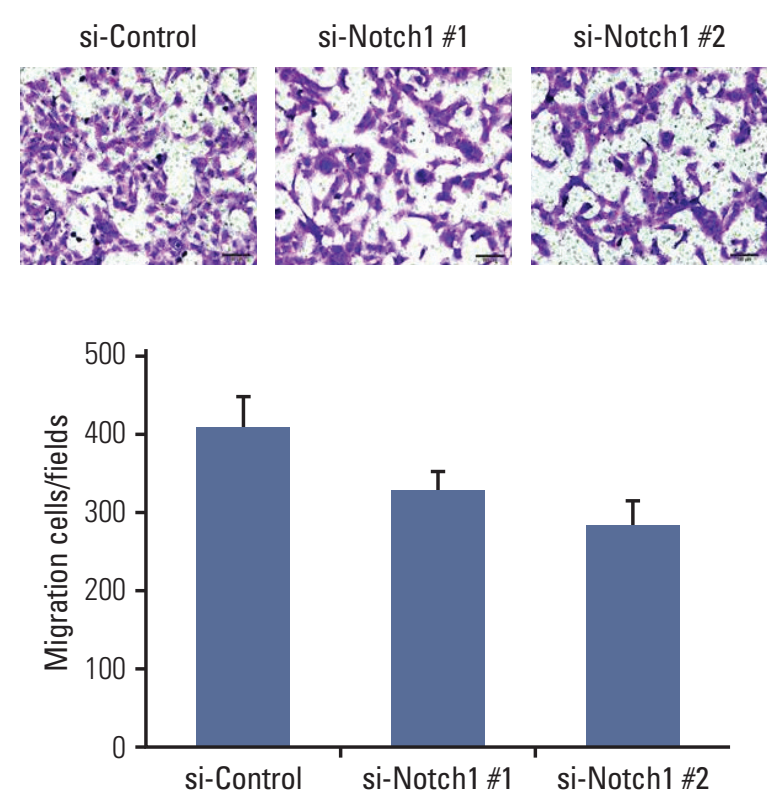

Fig. 3. Knockdown of Notch1 inhibited cell migration in anaplastic thyroid cancer cell lines. For the analysis of cell migration, 8505C (A) and BCPAP (B) cells in which Notch1 was knocked down were plated into transwell inserts, and 10\% fetal bovine serum was used in the medium in the bottom chambers. After 48 hours, migration of thyroid cancer cells was quantified $(\times 100)$. Statistical significance is based on the difference when compared with si-control cells, ${ }^{* *} \mathrm{p}<0.01,{ }^{* * *} \mathrm{p}<0.001$.

\section{Knockdown of Notch1 inhibited cell migration in ATC cell lines}

We examined the effect of Notch1 on cell motility using a transwell migration assay. $8505 \mathrm{C}$ and BCPAP cells were transfected with Notch1 siRNA at $50 \mathrm{nM}$ for 72 hours. As shown in Fig. 3, knockdown of Notch1 dramatically suppressed the migration ability of $8505 \mathrm{C}$ cells, but not that of BCPAP cells. These results suggested that knockdown of Notch1 inhibited the cell migration ability of ATC cells.

\section{DAPT reduced cell viability and cell migration in ATC cell lines}

We determined the effects of DAPT, a $\gamma$-secretase inhibitor, on cell viability and cell migration. 8505C and BCPAP cells were treated with various concentrations $(0,2$, or $5 \mu \mathrm{M})$ of DAPT for 72 hours. We found that knockdown of Notch1 signaling by $5 \mu \mathrm{M}$ DAPT reduced the cell viability of $8505 \mathrm{C}$ cells, but not BCPAP cells (Fig. 4A). We also examined the effects of DAPT treatment on the migration of $8505 \mathrm{C}$ and BCPAP cells. Inhibition of Notch1 signaling decreased the number of migrating $8505 \mathrm{C}$ cells compared with the control, but BCPAP cells did not (Fig. 4B). These data indicate that Notch inhibitor suppressed growth and cell migration in ATC cells.

\section{Knockdown of Notch1 attenuated CSC- and EMT-related markers}

We analyzed the levels of CSC- and EMT-related proteins after knockdown of Notch1. We found that knockdown of Notch1 reduced the levels of proteins related to CSC and EMT, including Notch1, Musashi, Sox2, Oct3/4, Nanog, Snail, and N-cadherin in $8505 \mathrm{C}$ cells. Moreover, Notch1 knockdown led to increased protein levels of E-cadherin (Fig. 5). However, these changes were not observed in BCPAP cells. 

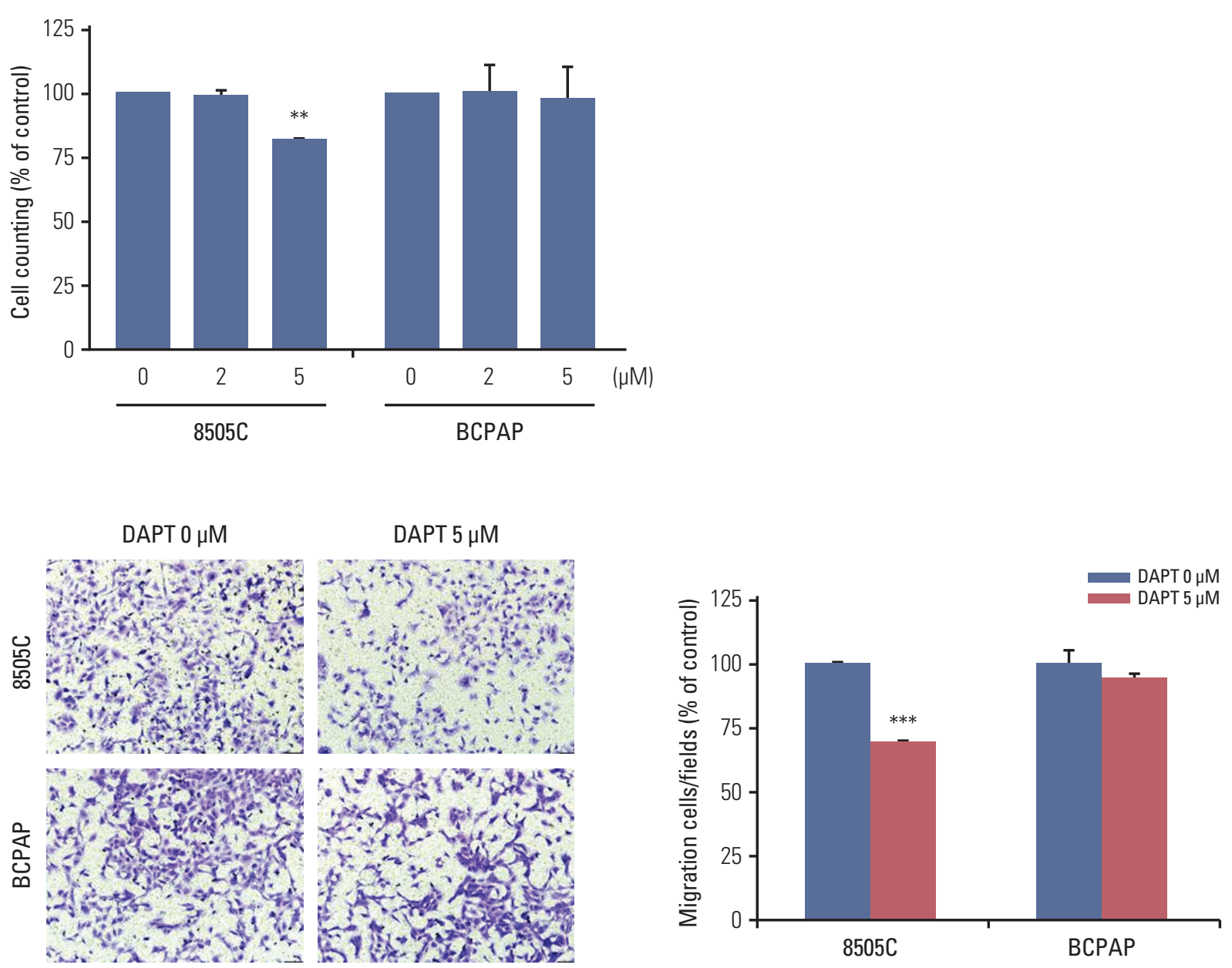

Fig. 4. DAPT reduced cell viability and cell migration in anaplastic thyroid cancer cell lines. (A) 8505C and BCPAP cells were treated with various concentrations of DAPT for 72 hours, after which proliferation was determined by cell counting. (B) The migration cells were stained with crystal violet and the images were photographed $(\times 100)$. Statistical significance is based on the difference when compared with untreated cells, ${ }^{* *} \mathrm{p}<0.01,{ }^{* * *} \mathrm{p}<0.001$. DAPT, $\mathrm{N}-[\mathrm{N}-(3,5$-difluorophenacetyl)L-alanyl]-S-phenylglycine $t$-butyl ester.

\section{Discussion}

High expression levels of Notch1 have been reported to play a major role in the metastasis of many cancer cells [19-21]. Studies of the role of Notch signaling in thyroid cancers appear to be highly context-dependent. The majority of studies of Notch signaling in thyroid cancers have shown it to be a tumor suppressor, especially in developed thyroid cancers such as PTC and follicular carcinomas (FC). For example, Ferreti et al. [22] and Xiao et al. [23] showed that Notch signaling was highly active in normal thyroid tissue, but decreased in developed thyroid cancers such as PTC and FC cells. Most of these studies analyzed well-developed thyroid cancer cells; therefore, investigations of the role of Notch signaling in poorly differentiated thyroid cancer, such as ATC, have been limited. Interestingly, Vasko et al. [24] demonstrated that the invasive front of PTC showed increased expression of EMT-related genes compared to the central lesion of PTC. The EMT process influences the conversion of early stage tumors to a stage of invasive malignancy and is closely related to tumor progression. In addition, it is well known that the EMT process regulates other signaling pathways, including Notch signaling. Based 

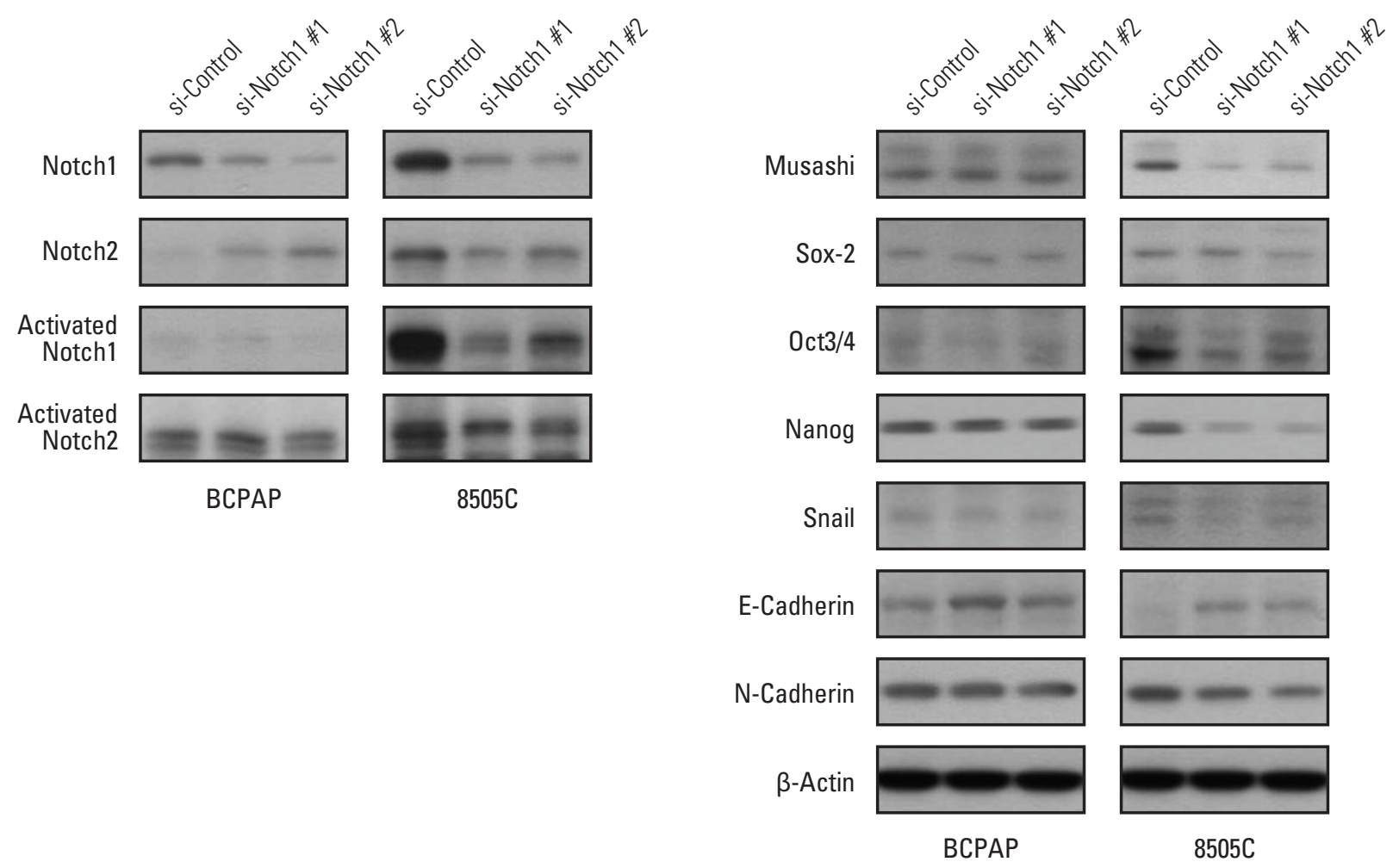

Fig. 5. Knockdown attenuated cancer stem cell (CSC)- and epithelial-mesenchymal transition (EMT)-related markers. 8505C and BCPAP cells were transfected with si-control or si-Notch1 for 72 hours. Total cell lysates were prepared, Notch1, Notch2, activated Notch1, activated Notch2 (A), and CSC- and EMT-related protein expression (B) were detected by western blot analysis.

on these results, we postulated that Notch signaling influences the malignant potential of thyroid cancers. To verify this hypothesis, we evaluated the different roles of Notch signaling in PTC and ATC. First, we checked the activation of Notch1 signaling in ATC and PTC. We observed increased expression of activated Notch1 in ATC patient tissues compared to PTC patient tissues. Immunohistochemical staining for activated Notch1, which indicates the activation of Notch1 signaling, showed that ATC patients had significantly higher expression than PTC patients. Additionally, ATC cells showed higher expression of Notch1 and activated Notch1 than PTC cells in vitro. Therefore, we concluded that Notch1 signaling was more active in ATC than PTC cells.

Subsequently, we compared the effects of knockdown of Notch1 signaling in ATC and PTC cells. We found that knockdown of Notch1 expression significantly increased the percentage of cell death and suppressed cell proliferation and migration in ATC cells. However, these results were not observed in PTC cells. Clinical aspects of ATC and PTC were entirely different; namely, ATC showed more rapid disease progression than PTC, including invasion to surrounding tissue and distant metastasis to other organs. Therefore, we concluded that activation of Notch1 signaling in ATC was an important factor influencing the clinical aspects of ATC through the increased cell proliferation or immortality and migration ability.

In the Notch signaling pathway, the extracellular domain of the Notch receptor binds to the ligands. This leads to cytoplasmic cleavage of the NICD by $\gamma$-secretase. NICD rapidly translocates to the nucleus and interacts with DNAbinding proteins to activate target genes $[8,12]$. Thus, Notch plays important roles in the regulation of cell growth, differentiation, development, and apoptosis $[25,26]$. Conventional treatments such as surgery, chemotherapy, and radiation therapy have not been effective in slowing the progression of ATC to date; accordingly, it is necessary to develop new therapeutic approaches, including targeted therapy, in ATC patient treatment. Our findings showed that DAPT, a $\gamma$-secretase inhibitor, effectively inhibited the proliferation and migration ability of ATC cells. Therefore, we concluded that 
DAPT could be a candidate for therapy that targets Notch signaling, perhaps presenting a novel therapeutic approach for ATC. Saad et al. [27] reported that Notch mediated EMT via increased expression of Snail, one of the major transcription factors involved in EMT. Notch promotes EMT via Snail induction and subsequent E-cadherin downregulation during normal tissue development and oncogenic transformation.

Notch1, which is involved in the regulation of not only EMT, but also the CSC phenotype during tumor progression, converts polarized epithelial cells into motile, invasive cells $[19,28,29]$. However, no data has demonstrated a direct relationship between Notch1 expression and the EMT and CSC phenotypes in thyroid cancers. Interestingly, we previously found that proteins related to EMT and CSC were more highly expressed in ATC than PTC tissues [30]. In addition, our results showed that knockdown of Notch1 expression decreased the expression of EMT- and CSC-related protein markers in ATC cells, but not in PTC cells. Based on these results, we hypothesized that this induction of EMT and CSC markers by activation of Notch1 signaling influenced the clinical behavior of ATC, which showed more malignant behavior than PTC. In addition, activation of Notch1 signaling is significantly associated with the proliferative activity or highly invasive nature of ATC cells regulating the EMT process and CSC features.

\section{Conclusion}

In summary, the present study shows that Notch1 inhibits proliferation and migration in ATC cells. In addition, knockdown of Notch1 expression reduced the expression of EMTrelated proteins and CSC markers in vitro. Overall, our data suggest that Notch1 is a possible target molecule for ATC treatment.

\section{Conflicts of Interest}

Conflict of interest relevant to this article was not reported.

\section{Acknowledgments}

This study was supported by a grant from the Korea Institute of Radiological and Medical Sciences (KIRAMS), funded by Ministry of Science, ICT and Future Planning, Republic of Korea $(1711031797 / 1711031800)$.

\section{References}

1. Jemal A, Siegel R, Xu J, Ward E. Cancer statistics, 2010. CA Cancer J Clin. 2010;60:277-300.

2. Nikiforov YE. Thyroid carcinoma: molecular pathways and therapeutic targets. Mod Pathol. 2008;21 Suppl 2:S37-43.

3. Neff RL, Farrar WB, Kloos RT, Burman KD. Anaplastic thyroid cancer. Endocrinol Metab Clin North Am. 2008;37:525-38.

4. Pasieka JL. Anaplastic thyroid cancer. Curr Opin Oncol. 2003;15:78-83.

5. Smallridge RC, Copland JA. Anaplastic thyroid carcinoma: pathogenesis and emerging therapies. Clin Oncol (R Coll Radiol). 2010;22:486-97.

6. Maillard I, Pear WS. Notch and cancer: best to avoid the ups and downs. Cancer Cell. 2003;3:203-5.

7. Yoon K, Gaiano N. Notch signaling in the mammalian central nervous system: insights from mouse mutants. Nat Neurosci. 2005;8:709-15.

8. Kopan R, Ilagan MX. The canonical Notch signaling pathway: unfolding the activation mechanism. Cell. 2009;137:216-33.

9. Wilson A, Radtke F. Multiple functions of Notch signaling in self-renewing organs and cancer. FEBS Lett. 2006;580:2860-8.

10. Bray SJ. Notch signalling: a simple pathway becomes complex.
Nat Rev Mol Cell Biol. 2006;7:678-89.

11. Kanwar R, Fortini ME. Notch signaling: a different sort makes the cut. Curr Biol. 2004;14:R1043-5.

12. Fortini ME. Gamma-secretase-mediated proteolysis in cell-surface-receptor signalling. Nat Rev Mol Cell Biol. 2002;3:673-84.

13. van Es JH, van Gijn ME, Riccio O, van den Born M, Vooijs M, Begthel $\mathrm{H}$, et al. Notch/gamma-secretase inhibition turns proliferative cells in intestinal crypts and adenomas into goblet cells. Nature. 2005;435:959-63.

14. Cao YW, Wan GX, Sun JP, Cui XB, Hu JM, Liang WH, et al. Implications of the Notch1-Snail/Slug-epithelial to mesenchymal transition axis for lymph node metastasis in infiltrating ductal carcinoma. Kaohsiung J Med Sci. 2015;31:70-6.

15. Gopalakrishnan N, Sivasithamparam ND, Devaraj H. Synergistic association of Notch and NFkappaB signaling and role of Notch signaling in modulating epithelial to mesenchymal transition in colorectal adenocarcinoma. Biochimie. 2014;107 Pt B:310-8.

16. Stylianou S, Clarke RB, Brennan K. Aberrant activation of notch signaling in human breast cancer. Cancer Res. 2006;66: 1517-25. 
17. Wang Z, Li Y, Kong D, Sarkar FH. The role of Notch signaling pathway in epithelial-mesenchymal transition (EMT) during development and tumor aggressiveness. Curr Drug Targets. 2010;11:745-51.

18. Bao B, Wang Z, Ali S, Kong D, Li Y, Ahmad A, et al. Notch-1 induces epithelial-mesenchymal transition consistent with cancer stem cell phenotype in pancreatic cancer cells. Cancer Lett. 2011;307:26-36.

19. Chen W, Cao G, Yuan X, Zhang X, Zhang Q, Zhu Y, et al. Notch-1 knockdown suppresses proliferation, migration and metastasis of salivary adenoid cystic carcinoma cells. J Transl Med. 2015;13:167.

20. Zhang Y, Li B, Ji ZZ, Zheng PS. Notch1 regulates the growth of human colon cancers. Cancer. 2010;116:5207-18.

21. Wang Z, Banerjee S, Li Y, Rahman KM, Zhang Y, Sarkar FH. Down-regulation of notch-1 inhibits invasion by inactivation of nuclear factor-kappaB, vascular endothelial growth factor, and matrix metalloproteinase-9 in pancreatic cancer cells. Cancer Res. 2006;66:2778-84.

22. Ferretti E, Tosi E, Po A, Scipioni A, Morisi R, Espinola MS, et al. Notch signaling is involved in expression of thyrocyte differentiation markers and is down-regulated in thyroid tumors. J Clin Endocrinol Metab. 2008;93:4080-7.

23. Xiao X, Ning L, Chen H. Notch1 mediates growth suppression of papillary and follicular thyroid cancer cells by histone deacetylase inhibitors. Mol Cancer Ther. 2009;8:350-6.

24. Vasko V, Espinosa AV, Scouten W, He H, Auer H, Liya- narachchi S, et al. Gene expression and functional evidence of epithelial-to-mesenchymal transition in papillary thyroid carcinoma invasion. Proc Natl Acad Sci U S A. 2007;104:2803-8.

25. Su BH, Qu J, Song M, Huang XY, Hu XM, Xie J, et al. NOTCH1 signaling contributes to cell growth, anti-apoptosis and metastasis in salivary adenoid cystic carcinoma. Oncotarget. 2014;5: 6885-95.

26. Miele L, Miao H, Nickoloff BJ. NOTCH signaling as a novel cancer therapeutic target. Curr Cancer Drug Targets. 2006;6: 313-23.

27. Saad S, Stanners SR, Yong R, Tang O, Pollock CA. Notch mediated epithelial to mesenchymal transformation is associated with increased expression of the Snail transcription factor. Int J Biochem Cell Biol. 2010;42:1115-22.

28. Leong KG, Niessen K, Kulic I, Raouf A, Eaves C, Pollet I, et al. Jagged1-mediated Notch activation induces epithelial-to-mesenchymal transition through Slug-induced repression of E-cadherin. J Exp Med. 2007;204:2935-48.

29. Buehler D, Hardin H, Shan W, Montemayor-Garcia C, Rush PS, Asioli S, et al. Expression of epithelial-mesenchymal transition regulators SNAI2 and TWIST1 in thyroid carcinomas. Mod Pathol. 2013;26:54-61.

30. Jung CW, Han KH, Seol H, Park S, Koh JS, Lee SS, et al. Expression of cancer stem cell markers and epithelial-mesenchymal transition-related factors in anaplastic thyroid carcinoma. Int J Clin Exp Pathol. 2015;8:560-8. 